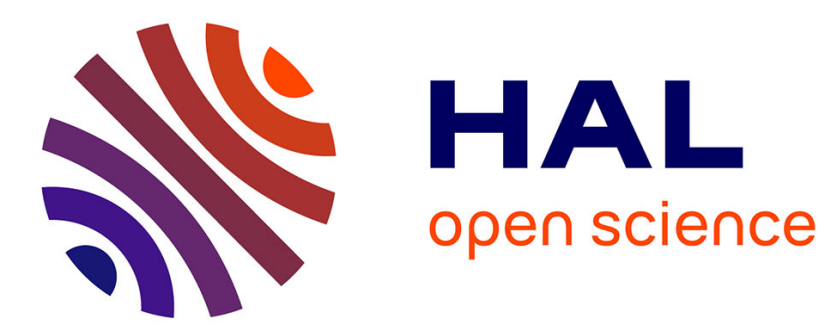

\title{
Narrowing Based Inductive Proof Search
}

Claude Kirchner, Helene Kirchner, Fabrice Nahon

\section{To cite this version:}

Claude Kirchner, Helene Kirchner, Fabrice Nahon. Narrowing Based Inductive Proof Search. 2011. hal-00692193

\section{HAL Id: hal-00692193 \\ https://hal.inria.fr/hal-00692193}

Submitted on 29 Apr 2012

HAL is a multi-disciplinary open access archive for the deposit and dissemination of scientific research documents, whether they are published or not. The documents may come from teaching and research institutions in France or abroad, or from public or private research centers.
L'archive ouverte pluridisciplinaire HAL, est destinée au dépôt et à la diffusion de documents scientifiques de niveau recherche, publiés ou non, émanant des établissements d'enseignement et de recherche français ou étrangers, des laboratoires publics ou privés. 


\title{
Narrowing Based Inductive Proof Search
}

\author{
Claude Kirchner ${ }^{1}$ and Hélène Kirchner ${ }^{1}$ and Fabrice Nahon ${ }^{2}$ \\ 1 INRIA France \\ first.last@inria.fr \\ 2 Rectorat Nancy-Metz, France \\ fabricenahon@googlemail.com
}

In memory of Harald Ganzinger

\begin{abstract}
We present in this paper a narrowing-based proof search method for inductive theorems. It has the specificity to be grounded on deduction modulo and to yield a direct translation from a successful proof search derivation to a proof in the sequent calculus. The method is shown to be sound and refutationally correct in a proof theoretical way. Keywords: deduction modulo, sequent calculus modulo, induction, Noetherian induction, induction by rewriting, equational reasoning, term rewriting
\end{abstract}

\section{Introduction}

Proof by induction is a main reasoning principle and is of prime interest in informatics and mathematics. Typically in hardware and software verification problems, reasoning on complex data structures with infinite data or states make a prominent use of induction and most deep mathematical theorem proofs rely on induction. Two main approaches have been developed for mechanizing induction proof: explicit induction, used in proof assistants, and implicit induction by rewriting, used in automated theorem provers. This work was motivated by the need to have a better understanding of the relation between them. Thanks to the deduction modulo framework, explicit induction is applied to generate smaller instances of the property to be proved. These instances can then be used by the modulo part to implicitly simplify the goals, thanks to a sequent calculus modulo.

In this context, we provide a proof search mechanism for such inductive proofs. We show how the induction step can be performed by narrowing at innermost positions when the theory is axiomatized by a sufficiently complete and convergent rewrite system. This allows us to make precise the relationship between rewrite-based automated inductive theorem provers like Spike or RRL and case analysis in proof assistants like Coq or PVS.

We provide a proof theoretic foundation to the proof search procedure which is described by deduction rules that are proved valid in the sequent calculus modulo. This provides the ability to build a proof term for a proof assistant and therefore to be able to formally validate the proof search result. So, starting from 
the (inductive) proposition to be proved, the proof search mechanism builds a proof in the sequent calculus modulo, from which a proof term can be computed if needed.

This paper was presented at the event organized in 2005 to celebrate Harald Ganzinger's memory and research contributions.

The paper is built over the works and results on deduction modulo [13], first-order presentation of higher-order logic [12], formalization of induction in deduction modulo $[7,8]$ and on preliminary results on narrowing for induction presented in [9]. We provide first a summary of these approaches in Section 1 to motivate the main idea of narrowing based induction proof search. Section 2 introduces two basic ingredients of the method: ordering on equalities and narrowing with sufficiently complete rewrite systems. Then Section 3 presents the proof search system for inductive proofs, which is proved sound and refutationally correct.

For the main notations and classical results on term rewriting, we refer to the books on that topics like [1] or [20].

\section{Deduction modulo and the Noetherian induction principle}

Proofs by structural induction are of main use in proof assistants where the structural induction principle is generally automatically generated from the definition of the inductive data types. However, by using sophisticated termination orderings, proofs by Noetherian induction performed by rewriting are much more expressive than structural induction. We recall in this section how deduction modulo can provide the description, at the proof theoretical level, of proof by Noetherian induction.

\subsection{Deduction modulo}

Let $\mathcal{T}(\Sigma, \mathcal{X})$ be the set of terms build over the signature $\Sigma$ and the denumerable set of variables $\mathcal{X}$. We assume for simplicity $\Sigma$ to be one-sorted, so that any term is of sort $\tau$. Terms are denoted by letters $s, t, u, v, l, r$, variables by $x, y, z, X, Y, Z$, vectors of variables by $\vec{x}$, and substitutions on terms by Greek letters $\alpha, \beta, \gamma$. Subst ${ }^{\mathcal{T}(\Sigma, \mathcal{X})}$ denotes the set of substitutions on $\mathcal{T}(\Sigma, \mathcal{X})$.

Provided a Noetherian relation $R$ and a user defined theory $T h_{u}$, we are looking for a proof of a proposition $P$ using a Noetherian induction principle denoted NoethInd, in the sense of finding a derivation of the sequent:

$$
\operatorname{NoethInd}(R), T h_{u} \vdash P
$$

The Noetherian induction principle being by essence a second order proposition, this is indeed a sequent in higher-order logic.

Since we want to make a primarily use of first-order rewrite concepts and techniques and to consider first-order theories, we need a first-order presentation 
of higher-order logic. We use the so-called $H O L_{\lambda \sigma}$ introduced in [12] which is based on deduction modulo [13] and reveals to be particularly well-suited for our concerns. It is clearly out of the scope of this paper to explain in detail the full approach, and we only sketch here the main ideas. The reader can refer to [7] and to $[8]$ for a detailed exposition.

In deduction modulo, terms but also propositions can be identified modulo a congruence. We use a congruence that can typically be defined by conditional equalities and that takes into account the application context to evaluate the conditions. Furthermore, since the congruence application should be controlled closely, an appropriate notion of protective symbol is used, see [7]: actually the congruence is not allowed to act below a protective symbol. In deduction modulo, the notions of term and proposition come from many-sorted first-order logic. We consider theories described by a set of axioms $\Gamma$ and a congruence, denoted $\sim$, defined on terms and propositions. This congruence takes three arguments: the two objects to be compared and a set of axioms $\Gamma$ called a local context. When we want to emphasize this, we denote the congruence $\sim^{\Gamma}$. The deduction rules of the sequent calculus take this equivalence into account. For instance, the right rule for the conjunction is not stated as usual

$$
\frac{\Gamma \vdash A, \Delta \quad \Gamma \vdash B, \Delta}{\Gamma \vdash A \wedge B, \Delta}
$$

but is formulated

$$
\frac{\Gamma \vdash A, \Delta \quad \Gamma \vdash \sim B, \Delta}{\Gamma \vdash \sim D, \Delta} \text { if } D \sim \Gamma A \wedge B .
$$

We recall in Figure 1, the definition of the sequent calculus modulo. In these rules, $\Gamma$ and $\Delta$ are finite multisets of propositions, $P$ and $Q$ denote propositions. Substituting the variable $x$ by the term $u$ in $Q$ is denoted $Q\{u / x\}$. When the congruence $\sim$ is simply identity, this sequent calculus collapses to the usual one [16]. In that case, sequents are written as usual with the $\vdash$ symbol.

Proof checking decidability for the sequent calculus modulo reduces to the decidability of the relation $\sim^{\Gamma}$, since we can check for each rule that the conditions of application are satisfied and we provide the needed information in the quantifier rules. When $\sim^{\Gamma}$ is not decidable, we still can use instances for which one can check the conditions of application, typically using a constraint based approach $[17,21]$

We can now introduce the fundamental notion of compatibility: a theory (a set of propositions) $\mathcal{T}$ is said to be compatible with a congruence $\sim$ when:

$$
\mathcal{T}, \Gamma \vdash \Delta \text { if and only if } \Gamma \vdash \Delta \text {. }
$$

As shown in $[7,8]$, this property is modular: if $\mathcal{T}_{1}$ is compatible with a congruence $C_{1}$ and $\mathcal{T}_{2}$ is compatible with $C_{2}$ then $\mathcal{T}_{1} \cup \mathcal{T}_{2}$ is compatible with $C_{1} \cup C_{2}$.

Using the above equivalence, we can internalize propositions into the congruence, and we call this operation "push". We can also recover them at the level 


$$
\begin{aligned}
& \overline{\Gamma, P \vdash Q} \text { axiom if } P \sim^{\Gamma} Q \\
& \frac{\Gamma, Q_{1}, Q_{2} \vdash \sim \Delta}{\Gamma, P \vdash \sim \Delta} \text { contr-l if }(\mathbf{A}) \\
& \frac{\Gamma, P \vdash \Delta \quad \Gamma \vdash \sim Q, \Delta}{\Gamma \vdash \sim} \text { cut if } P \sim^{\Gamma} Q \\
& \frac{\Gamma \vdash \Delta}{\Gamma, P \vdash \sim \Delta} \text { weak-l } \\
& \frac{\Gamma \vdash \sim Q_{1}, Q_{2}, \Delta}{\Gamma \vdash \sim P, \Delta} \text { contr-r if }(\mathbf{A}) \\
& \frac{\Gamma, P, Q \vdash \Delta}{\Gamma, R \vdash \Delta} \wedge-1 \text { if } R \sim^{\Gamma}(P \wedge Q) \\
& \frac{\Gamma \vdash \Delta}{\Gamma \vdash \sim P, \Delta} \text { weak-r } \\
& \frac{\Gamma, P \vdash \Delta \quad \Gamma, Q \vdash \sim}{\Gamma, R \vdash \sim} \vee \text {-l if }(\mathbf{B}) \\
& \frac{\Gamma \vdash \sim P, \Delta \quad \Gamma \vdash \sim Q, \Delta}{\Gamma \vdash R, \Delta} \wedge \text { if } R \sim^{\Gamma}(P \wedge Q) \\
& \frac{\Gamma \vdash P, \Delta \quad \Gamma, Q \vdash \Delta}{\Gamma, R \vdash \sim} \Rightarrow-1 \text { if }(\mathbf{C}) \\
& \frac{\Gamma \vdash P, Q, \Delta}{\Gamma \vdash R, \Delta} \vee \text {-r if }(\mathbf{B}) \\
& \frac{\Gamma \vdash P, \Delta}{\Gamma, R \vdash \sim \Delta} \neg-1 \text { if } R \sim^{\Gamma} \neg P \\
& \frac{\Gamma, P \vdash Q, \Delta}{\Gamma \vdash R, \Delta} \Rightarrow-\mathrm{r} \quad \text { if }(\mathbf{C}) \\
& \overline{\Gamma, P \vdash \Delta} \perp-1 \quad \text { if } P \sim^{\Gamma} \perp \\
& \frac{\Gamma, Q\{t / x\} \vdash \sim \Delta}{\Gamma, P \vdash \sim \Delta}(Q, x, t) \forall-1 \text { if }(\mathbf{D}) \\
& \frac{\Gamma, P \vdash \sim \Delta}{\Gamma \vdash R, \Delta} \neg \text {-r if } R \sim^{\Gamma} \neg P \\
& \frac{\Gamma, Q\{y / x\} \vdash \Delta}{\Gamma, P \vdash \sim}(Q, x, y) \exists-\mathrm{l} \quad \text { if }(\mathbf{F}) \\
& \frac{\Gamma \vdash \sim\{y / x\}, \Delta}{\Gamma \vdash \sim P, \Delta}(Q, x, y) \forall-\mathrm{r} \text { if }(\mathbf{E}) \\
& \frac{\Gamma \vdash_{\sim} Q\{t / x\}, \Delta}{\Gamma \vdash \sim P, \Delta}(Q, x, t) \exists-\mathrm{r} \quad \text { if }(\mathbf{G}) \\
& \mathbf{A}=P \sim^{\Gamma} Q_{1} \sim^{\Gamma} Q_{2}, \mathbf{B}=R \sim^{\Gamma}(P \vee Q) \mathbf{C}=R \sim^{\Gamma}(P \Rightarrow Q), \mathbf{D}=P \sim^{\Gamma} \forall x Q, \\
& \mathbf{E}=P \sim^{\Gamma} \forall x Q, y \text { fresh variable, } \mathbf{F}=P \sim^{\Gamma} \exists x Q, y \text { fresh variable, } \mathbf{G}=P \sim^{\Gamma} \exists x Q
\end{aligned}
$$

Fig. 1. The sequent calculus modulo

of the logic, and we call this operation "pop". Moreover, thanks to modularity, this can be done dynamically during the proof. This duality between computation and deduction is very conveniently reflected by the compatibility property. In [13], internalization has been done statically and used to identify computation within the deduction process. Our aim here is to do internalization dynamically and to use it to design rules for induction by rewriting and an adequate strategy for Noetherian induction.

In what follows, we consider congruences generated by conditional class rewrite systems denoted $\mathcal{R} \mathcal{E}$ and composed of (conditional) term rewrite rules, (conditional) term equational axioms, (conditional) proposition rewrite rules, (conditional) proposition equational axioms. Moreover, we assume that the lefthand side of a proposition rewrite rule and both sides of a proposition equational axiom have to be atomic propositions. Conditions may be arbitrary propositions. The variables in the right-hand side and condition of a rule must occur in the left-hand side. In the case of equational axioms, variables in both sides have to be the same and (free) variables in the condition have to be a subset of those. We assume here that $\approx$ is a binary relation symbol which satisfies the axioms of equality (the classical denotation $=$ will only represent syntactical equality). In this case, to any conditional class rewrite system $\mathcal{R E}$ is associated the theory de- 
noted $T_{\mathcal{R E}}$ as follows: for each conditional rewrite rule $(l \rightarrow r$ if $c)$ or conditional equality $\left(l \approx r\right.$ if $c$ ) in $\mathcal{R E}, T_{\mathcal{R E}}$ contains the proposition:

$-\forall \bar{x}(c \Rightarrow(l \Leftrightarrow r))$ when $l$ and $r$ are propositions,

$-\forall \bar{x}(c \Rightarrow(l \approx r))$ when $l$ and $r$ are terms,

where all free variables of $l$, denoted $\bar{x}$, are universally quantified.

It is proved in [7] that $T_{\mathcal{R E}}$ is compatible with the congruence generated by $\mathcal{R E}$ (see also [11] and [13]). This allows us to freely use the "pushing and popping" operations. This also ensures that deduction modulo a congruence represented by a conditional class rewrite system is not a proper extension of first-order logic, but only a different presentation of it.

\subsection{Deduction modulo for inductive proofs}

This short introduction to deduction modulo now allows us to give a proof theoretic understanding of induction by rewriting. In the context of deduction modulo, the induction hypotheses arising from equational goals can be (dynamically) internalized into the congruence. When doing this, the computational part of the deduction modulo appears to perform induction by rewriting as done for instance by systems like Spike [4] or RRL [19].

The powerful principle of these approaches is to allow application of rewrite rules of the theory at any position of the current goal, as well as application of induction hypotheses and current conjecture, provided that the applied formula is smaller in the Noetherian induction ordering than the current goal.

When the ordering contains the relation induced by a terminating rewrite system, a smaller formula is obtained as soon as a rewrite step is performed. Moreover, in Spike for instance, the choice of the induction variables and instantiation schemas is done using pre-calculated induction positions and schemas called test-sets. In the approach described below, we show how to use narrowing to automatically and completely perform these choices. The whole problem is formalized in $H O L_{\lambda \sigma}$.

Given a property $P$ and a relation $R$ defined on a sort $\tau$, the Noetherian induction principle $\operatorname{Noeth} \operatorname{Ind}(P, R, \tau)$ is defined as follows:

$$
\forall x((x \in \tau \wedge \forall y((y \in \tau \wedge R(y, x)) \Rightarrow P(y))) \Rightarrow P(x)) \Rightarrow \forall x(x \in \tau \Rightarrow P(x))
$$

and we write $\operatorname{Noeth}(R, \tau)$ to state that $R$ is a Noetherian relation over $\tau$.

Proving that $P$ inductively holds in a user theory $T h_{u}$, denoted $T h_{u} \models_{\text {Ind }} P$, amounts to derive the sequent:

$$
\forall R \forall \tau(\operatorname{Noeth}(R, \tau) \Rightarrow \forall P \operatorname{NoethInd}(P, R, \tau)), T h_{u} \vdash P .
$$

Of course to finish the proof, one should also provide a proof of $N$ oeth $(R, \tau)$. To get a better intuition, let us consider an equational goal $Q$ of the form $\forall x(x \in$ 
$\left.\tau \Rightarrow t_{1}(x) \approx t_{2}(x)\right)$. The remainder of this section gives the main steps which are detailed in [7]. We start from the sequent:

$$
\begin{aligned}
& \forall R \forall \tau(\operatorname{Noeth}(R, \tau) \Rightarrow \forall P \operatorname{NoethInd}(P, R, \tau)), T h_{u} \\
& \vdash \\
& \forall x\left(x \in \tau \Rightarrow t_{1}(x) \approx t_{2}(x)\right)
\end{aligned}
$$

In the following, we will denote $N I$ the proposition:

$$
\forall R \forall \tau(\operatorname{Noeth}(R, \tau) \Rightarrow \forall P \operatorname{NoethInd}(P, R, \tau))
$$

Choosing a specific relation $R$ (written $\prec$ ) and a type still denoted $\tau$, we get:

$$
\operatorname{Noeth}(\prec, \tau) \Rightarrow \forall P \operatorname{NoethInd}(P, \prec, \tau)), T h_{u} \vdash \forall x\left(x \in \tau \Rightarrow t_{1}(x) \approx t_{2}(x)\right) \text {. }
$$

From this, by the rule $\Rightarrow-1$ of the sequent calculus, we get on one hand the sequent $T h_{u} \vdash \operatorname{Noeth}(\prec, \tau)$ corresponding to the proof that $\prec$ is indeed Noetherian, on the other hand the sequent

$$
\forall P \operatorname{NoethInd}(P, \prec, \tau)), T h_{u} \vdash \forall x\left(x \in \tau \Rightarrow t_{1}(x) \approx t_{2}(x)\right)
$$

corresponding to the use of the induction principle to prove our goal.

We instantiate $P$ as the equality to prove and we get:

$$
\begin{aligned}
& \forall x\left(\left(x \in \tau \wedge \forall \underline{x}\left((\underline{x} \in \tau \wedge \underline{x} \prec x) \Rightarrow t_{1}(\underline{x}) \approx t_{2}(\underline{x})\right)\right) \Rightarrow t_{1}(x) \approx t_{2}(x)\right) \\
& \Rightarrow \forall x\left(x \in \tau \Rightarrow t_{1}(x) \approx t_{2}(x)\right), T h_{u} \vdash \forall x\left(x \in \tau \Rightarrow t_{1}(x) \approx t_{2}(x)\right)
\end{aligned}
$$

where we have renamed $y$ to $\underline{x}$ to emphasize that $\underline{x}$ is a smaller instance of $x$. A few easy steps of the sequent calculus later, we get:

$$
T h_{u} \vdash \forall x\left(\left(x \in \tau \wedge \forall \underline{x}\left((\underline{x} \in \tau \wedge \underline{x} \prec x) \Rightarrow t_{1}(\underline{x}) \approx t_{2}(\underline{x})\right)\right) \Rightarrow t_{1}(x) \approx t_{2}(x)\right)
$$

We then instantiate $x$ by a fresh variable that we call $X$ to emphasize this status, and we get:

$$
T h_{u} \vdash\left(X \in \tau \wedge \forall \underline{x}\left((\underline{x} \in \tau \wedge \underline{x} \prec X) \Rightarrow t_{1}(\underline{x}) \approx t_{2}(\underline{x})\right)\right) \Rightarrow t_{1}(X) \approx t_{2}(X) .
$$

The $\Rightarrow-\mathrm{r}$ and $\wedge-\mathrm{l}$ rules of the sequent calculus lead to the discovery of the induction hypothesis:

$$
\left.T h_{u}, X \in \tau, \forall \underline{x}\left((\underline{x} \in \tau \wedge \underline{x} \prec X) \Rightarrow t_{1}(\underline{x}) \approx t_{2}(\underline{x})\right)\right) \vdash t_{1}(X) \approx t_{2}(X) .
$$

Using what we have seen on compatible theories, this hypothesis can now be internalized as a conditional equality denoted in general $\mathcal{R E}_{\text {ind }}(Q, \prec, \tau)(X)$ :

$$
t_{1}(\underline{x}) \approx t_{2}(\underline{x}) \text { if } \underline{x} \in \tau \wedge \underline{x} \prec X
$$

Note that because of its status of free fresh variable, $X$ behaves like a constant, while $\underline{x}$ is universally quantified.

What is crucial in using the induction hypothesis (1) as an equality or a rewrite rule, is to check its condition. For any many-sorted theory, the $\underline{x} \in \tau$ part of the condition just expresses that the variable is sorted.

One of the main technical point handled in the paper is to justify that in most cases, the condition $\underline{x} \prec X$ is easily checked when an induction hypothesis like (1) is internalized and used as a simplification rewrite rule. 


\section{Ordering and narrowing}

Before describing the proof search system, we describe in this section the two main tools of the method, namely orderings on terms and equalities, and the narrowing properties in sufficiently complete rewrite systems. Most importantly, we provide the main result (Theorem 1) relating induction as deduction modulo as presented in the previous section and the Noetherian ordering induced by a terminating rewrite relation.

\subsection{Orders and quasi-orders on terms and equalities}

The set of positions in a term $t$ is denoted $\operatorname{Dom}(t)$, the subterm of $t$ at position $\omega$ is denoted $t_{\mid \omega}$ and the symbol at position $\omega$ in $t$ by $t(\omega)$. The notation $t[u]_{\omega}$ means that the term $t$ contains the subterm $u$ at position $\omega$. These notations extend to goals $t_{1} \approx t_{2}$ seen as a term with top symbol $\approx$ of arity 2 . $\mathcal{V}$ ar $(t)$ denotes the set of (free) variables of the term $t$ and $|\mathcal{V} a r(t)|$ its cardinality. We define $\overrightarrow{\mathcal{V} a r(t)}$ as the vector of variables assumed linearly ordered by their name. These notations are extended to equalities, rewrite rules and goals.

From now on, we assume given a quasi simplification order $\leqslant$ on $\mathcal{T}(\Sigma, \mathcal{X})$ (see for example [10]). We denote $<$ its proper part, $\gtrless$ its associated equivalence (i.e. $\gtrless=(\leqslant \cap \geqslant)$ ) and $[t]$ the class of a term $t$ for this equivalence. We assume that $<$ and $\gtrless$ are closed under substitutions and contexts. For instance, it is shown in [14] that if $\leqslant$ is a recursive path ordering (rpo) with status then $<$ and $\gtrless$ are closed under substitutions and contexts.

In order to compare $n$-tuple of terms, for any natural $n$, we will use the standard extension on the Cartesian product $\leqslant n$ of $\leqslant$ :

$$
\forall \vec{u}, \vec{v} \in \mathcal{T}(\Sigma, \mathcal{X})^{n} \quad \vec{u} \leqslant_{n} \vec{v} \Leftrightarrow\left(\forall i 1 \leq i \leq n \Rightarrow u_{i} \leqslant v_{i}\right)
$$

If we denote $<_{n}$ the proper part of this quasi-order, then $<_{n}$ is Noetherian on the set $\mathcal{T}(\Sigma, \mathcal{X})^{n}$ provided $<$ is Noetherian. We extend this quasi-order to equalities in the following way:

$$
s \approx t \leqslant 2 s^{\prime} \approx t^{\prime} \text { if } s \leqslant s^{\prime} \text { and } t \leqslant t^{\prime} .
$$

Definition 1. Let $Q$ and $Q^{\prime}$ be two equational goals, $Q^{\prime} \leq_{e} Q$ whenever there exists a finite sequence of equalities $\left(Q_{i}=s_{i} \approx t_{i}\right)_{0 \leq i \leq n}$ such that:

1. $Q=Q_{0}$ and $Q^{\prime}=Q_{n}$,

2. for any $i, s_{i+1} \leqslant s_{i}$ and $t_{i}=t_{i+1}$ or $t_{i+1} \leqslant t_{i}$ and $s_{i}=s_{i+1}$.

Now, since $\leqslant$ is stable under substitution, we get:

Lemma $\mathbf{1} . \leq_{e}$ is stable under substitution.

Moreover, to compare goals in a finer way, we also will make use of another ordering on goals similar to the one in [7]. 
Definition 2. Let $C$ be the following complexity measure on equalities, where $\{[t]\}$ denotes the multiset of terms in the equivalence class of $t$ for $\gtrless$.

$$
\begin{aligned}
& C(s \approx t)=(\{[s]\},\{[t]\}) \text { if }[t]<[s] \\
& (\{[t]\},\{[s]\}) \text { if } \quad[s]<[t] \\
& (\{[s],[t]\}, \emptyset) \text { otherwise }
\end{aligned}
$$

We define a quasi ordering on equalities $\leqslant_{e}$ by

$$
s \approx t \leqslant_{e} s^{\prime} \approx t^{\prime} \text { if } C(s \approx t) \ll_{\text {lex }} C\left(s^{\prime} \approx t^{\prime}\right) \text { or }\left(s \gtrless s^{\prime} \text { and } t \gtrless t^{\prime}\right)
$$

where $\ll_{l e x}$ is the lexicographic extension of the multiset extension of $<$. We denote $<_{e}$ the proper part of $\leqslant_{e}$.

Let us remark that the order $<_{e}$ is well-suited for equalities, since it is invariant under symmetry of equality: for all $t, t^{\prime}, u, u^{\prime} \in \mathcal{T}(\Sigma, \mathcal{X})$, we have:

$t \approx t^{\prime}<_{e} u \approx u^{\prime}$ if and only if $t^{\prime} \approx t<_{e} u \approx u^{\prime}$ if and only if $t \approx t^{\prime}<_{e} u^{\prime} \approx u$. But it is not stable under substitution: for example with the substitution $\sigma=\left\{x \mapsto x_{1}, y \mapsto x_{1}, z \mapsto z_{1}\right\}$, we have:

1. $z \approx x+z<_{e} y \approx x+z$ since

$$
\begin{aligned}
& C(z \approx x+z)=(\{[x+z]\},\{[z]\}) \text { and } \\
& C(y \approx x+z)=(\{[x+z],[y]\}, \emptyset)
\end{aligned}
$$

2. but $z \sigma \approx x \sigma+z \sigma \Varangle_{e} y \sigma \approx x \sigma+z \sigma$ since

$C(z \sigma \approx x \sigma+z \sigma)=\left(\left\{\left[x_{1}+z_{1}\right]\right\},\left\{\left[z_{1}\right]\right\}\right)$ and

$C(y \sigma \approx x \sigma+z \sigma)=\left(\left\{\left[x_{1}+z_{1}\right]\right\},\left\{\left[x_{1}\right]\right\}\right)$

Notice the difference between $\leqslant_{e}$ and $\leq_{e}$, the latter being included in the former as it can be checked by a simple case analysis. Indeed, stability by substitution is in particular needed when considering optimized version of the proof search method developed in [23].

\subsection{Induction hypothesis and ordering on goals}

Taking into account vectors of variables, we are now in position to instantiate the Noetherian induction hypothesis $\mathcal{R E}_{\text {ind }}(Q, \prec, \tau)(X)$ defined in Section 1.2.

For any equality $Q$, for any integer $n$ such that $n=\mid \mathcal{V}$ ar $(Q) \mid$, for any $\vec{x} \in \mathcal{X}^{n}$ such that $\vec{x}$ is the vector of variables of $Q$, we have:

$$
\mathcal{R E}_{\mathcal{E}_{\text {ind }}}\left(Q,<_{n}, \mathcal{T}(\Sigma)^{n}\right) \triangleq\left(\underline{\vec{x}} \in \mathcal{T}(\Sigma)^{n}\right) \wedge\left(\underline{\vec{x}}<_{n} \vec{x}\right) \Rightarrow Q\{\underline{\vec{x}} / \vec{x}\}
$$

In order to simplify the notations, and when no confusion can occur, we denote it simply $\mathcal{R E}_{\text {ind }}(Q,<)$.

In the same way, we introduce the following notations, where $\sigma$ is any substitution:

$$
\begin{aligned}
& -\mathcal{R} \mathcal{E}_{\text {ind }}(Q,<) \sigma \triangleq\left(\underline{\vec{x}} \in \mathcal{T}(\Sigma)^{n}\right) \wedge\left(\underline{\vec{x}}<_{n} \vec{x} \sigma\right) \Rightarrow Q\{\underline{\vec{x}} / \vec{x}\} \\
& -\mathcal{R} \mathcal{E}_{\text {ind }}(Q, \leqslant) \triangleq\left(\underline{\vec{x}} \in \mathcal{T}(\Sigma)^{n}\right) \wedge(\underline{\vec{x}} \leqslant n \vec{x}) \Rightarrow Q\{\underline{\vec{x}} / \vec{x}\}
\end{aligned}
$$




$$
-\mathcal{R} \mathcal{E}_{\text {ind }}(Q, \leqslant) \sigma \triangleq\left(\underline{\vec{x}} \in \mathcal{T}(\Sigma)^{n}\right) \wedge(\underline{\vec{x}} \leqslant n \vec{x} \sigma) \Rightarrow Q\{\underline{\vec{x}} / \vec{x}\}
$$

A crucial point in inductive proofs will be to compare different instances of a same equational goal: this is the purpose of the next lemma.

Lemma 2. For any equational goal $Q$ with $\vec{x}=\overrightarrow{\mathcal{V} a r(Q)}$ and $n=|\vec{x}|$, for all substitutions $\sigma, \mu \in \operatorname{Subst}^{\mathcal{T}(\Sigma, \mathcal{X})}$, for all $t, t^{\prime} \in \mathcal{T}(\Sigma, \mathcal{X})$ :

1. If $t \leqslant t^{\prime}$ then $Q[t]_{\omega} \leqslant e\left[t^{\prime}\right]_{\omega}$

2. If $\vec{x} \sigma \leqslant n \vec{x} \mu$ then $Q \sigma \leqslant_{e} Q \mu$.

3. If $Q \sigma<_{e} Q \mu$ and $\vec{x} \sigma \leqslant_{n} \vec{x} \mu$ then $\vec{x} \sigma<_{n} \vec{x} \mu$.

Proof. 1. Let $i$ and $\omega^{\prime}$, such that $\omega=i \cdot \omega^{\prime}$ ( $i=1,2$ since $Q$ is an equality). Since $t \leqslant t^{\prime}$, and since $\leqslant$ is a reduction ordering, we have:

$$
Q_{\mid i}[t]_{\omega^{\prime}} \leqslant Q_{\mid i}\left[t^{\prime}\right]_{\omega^{\prime}}
$$

Now, one can easily check the following proposition:

$$
\forall s \forall s^{\prime} \forall u s \leqslant s^{\prime} \Rightarrow s \approx u \leqslant_{e} s^{\prime} \approx u
$$

And (2) and (3) above lead to $Q[t]_{\omega} \leqslant_{e} Q\left[t^{\prime}\right]_{\omega}$

2 . is obtained from 1 by an easy induction based on the number of occurrences of the variables $x_{i}$ in $Q$

3. Assume $\vec{x} \sigma \leqslant_{n} \vec{x} \mu$ and $\vec{x} \sigma \nless_{n} \vec{x} \mu$. Then $\vec{x} \sigma \gtrless_{n} \vec{x} \mu$, hence $\vec{x} \mu \leqslant_{n}$ $\vec{x} \sigma$, thus $Q \mu \leqslant_{e} Q \sigma$ by 2 , and this contradicts the assumption $Q \sigma<_{e}$ $Q \mu$.

In other words, for any equational goal $Q$, for any vector of variables $\vec{x}$ of $Q$ in $\mathcal{X}^{n}$, and for all $\sigma, \mu \in S u b s t^{\mathcal{T}(\Sigma, \mathcal{X})}$, in order to prove the proposition $\vec{x} \sigma<_{n} \vec{x} \mu$, and whenever $Q \sigma<_{e} Q \mu$, it suffices to check all inequalities $\sigma\left(x_{i}\right) \leqslant \mu\left(x_{i}\right)$ for all component $x_{i}$ of $\vec{x}$. Indeed, we are going to see in next Lemma that the inequality $Q \sigma<_{e} Q \mu$ can be automatically checked in many cases.

The next theorem relates the strict ordering $<_{e}$ on goals with a rewrite relation $\rightarrow$. It is a crucial step to justify the correct use of Noetherian rewriting as the main ingredient to perform Noetherian induction.

Indeed, under technical conditions that can be syntactically checked, this result ensures that $Q \sigma<_{e} Q \mu$. It is therefore possible in most of the cases to use an equational goal $Q$ to reduce an instance of itself, $Q \mu$, as soon as a rewrite step has been previously performed on $Q \mu$.

Theorem 1 (Main compatibility theorem). Let $Q_{1}, Q_{2}, Q_{3}$ and $Q_{4}$ be equational goals, $l \rightarrow r$ a rewrite rule (thus $l>r$ ), $\kappa_{0}$ be either a rewrite rule $l_{\kappa_{0}} \rightarrow r_{\kappa_{0}}$ or an equality $l_{\kappa_{0}} \approx r_{\kappa_{0}}$.

Let us consider the inequality $I:\left(l_{\kappa_{0}} \approx r_{\kappa_{0}}\right) \sigma<_{e} Q_{1}$ and assume:

$$
\text { 1. } Q_{1} \rightarrow_{l \rightarrow r, j . \omega_{j}, \theta} Q_{2}
$$


2. $Q_{2} \geqslant{ }_{2} Q_{3}$.

3. $Q_{3} \rightarrow_{\kappa_{0}, i . \omega_{i}, \sigma} Q_{4}$

4. $Q_{3} \geqslant_{e} Q_{4}$

Then:

1. I is satisfied whenever $\omega_{i} \neq \varepsilon$ or $i=j$

2. If $\omega_{i}=\varepsilon$ and $i \neq j$ :

(a) If $l_{\kappa_{0}}>r_{\kappa_{0}}$, then:

$$
I \Leftrightarrow\left(\left(Q_{1_{\mid i}} \gtrless l_{\kappa_{0}} \sigma\right) \wedge\left(Q_{1_{\mid j}}<Q_{1_{\mid i}}\right) \Rightarrow\left(Q_{1_{\mid j}}>r_{\kappa_{0}} \sigma\right)\right)
$$

(b) If $l_{\kappa_{0}} \gtrless r_{\kappa_{0}}$, then:

$$
I \Leftrightarrow\left(\left(Q_{1 \mid i} \gtrless l_{\kappa_{0}} \sigma\right) \Rightarrow\left(Q_{1 \mid j}>r_{\kappa_{0}} \sigma\right)\right)
$$

(c) Otherwise:

$$
\begin{aligned}
& I \Leftrightarrow\left(\left(\left(Q_{1 \mid i} \gtrless l_{\kappa_{0}} \sigma\right) \wedge\left(\left(Q_{1 \mid j}<Q_{1 \mid i}\right) \vee\left(l_{\kappa_{0}} \sigma \gtrless r_{\kappa_{0}} \sigma\right)\right) \wedge\left(r_{\kappa_{0}} \sigma \leqslant l_{\kappa_{0}} \sigma\right)\right)\right. \\
& \left.\quad \Rightarrow\left(Q_{1 \mid j}>r_{\kappa_{0}} \sigma\right)\right)
\end{aligned}
$$

Proof. The proof of this crucial result is given in [23]. It is based on a technical case analysis.

A variant of this theorem is given in [7] for an ordering between goals based on a complexity $C$ using a set ordering instead of multiset ordering as here.

\subsection{Narrowing}

To make precise the use of narrowing in the induction process, let us first introduce a few concepts and notations. Narrowing will be performed only with rewrite rules, i.e. formulas $l \rightarrow r$ with $l>r$, but not with equalities. Let $\mathcal{R}$ be a rewrite system on $\mathcal{T}(\Sigma, \mathcal{X})$. The signature $\Sigma$ is partitioned into a set of constructors $\mathcal{C}$ and a set of defined symbols $\mathcal{D}$. Constructors are function symbols which do not occur as a head symbol of a rule left-hand side. A constructor term is a term built only with constructor symbols. $\mathcal{T}(\mathcal{C}, \mathcal{X})$ denotes the set of constructor terms. A ground substitution is a substitution mapping each variable to a ground term, i.e. a term without variables. Let $S u b s t^{\mathcal{T}(\Sigma)}$ be the set of all ground substitutions on $\mathcal{T}(\Sigma)$. A rewrite system is said to be ground convergent if it is confluent and terminating over the set of ground terms. For any ground convergent rewrite system $\mathcal{R}$, a term $t$ is ground $\mathcal{R}$-reducible if $t \alpha$ is $\mathcal{R}$-reducible for any ground substitution $\alpha$. Furthermore, a symbol $f \in \mathcal{D}$ of arity $n$ is completely defined if $f\left(x_{1}, \ldots, x_{n}\right)$ is ground reducible, and a ground convergent rewrite system $\mathcal{R}$ is said to be sufficiently complete if all symbols in $\mathcal{D}$ are completely defined.

For ground convergent and sufficiently complete rewrite systems, it is possible to specify particular positions in terms where reductions must apply, and where case analysis by rewriting can usefully be done. 
Definition 3. For any $t \in \mathcal{T}(\Sigma, \mathcal{X})$, a position $\omega$ in $t$ is called defined-innermost, and we denote $\omega \in D I(t)$, if $t(\omega) \in \mathcal{D}$ and $t\left(\omega^{\prime}\right) \in \mathcal{C} \cup \mathcal{X}$ whenever $\omega<\omega^{\prime}$.

For example, considering Peano's naturals, 0 and $s$ are constructors, + is a defined symbol and in $s((0+0)+s(0+s(x)))$ the occurrence 1.2.1 is definedinnermost but 1 is not.

Lemma 3. For any ground convergent rewrite system $\mathcal{R}$, for any term $t$, and for any position $\omega \in \operatorname{Dom}(t)$, if $t(\omega)$ is completely defined and $\omega$ is defined-innermost in $\operatorname{Dom}(t)$, then, for any irreducible ground substitution $\alpha, t \alpha$ is reducible at position $\omega$.

Proof. Classical and by case analysis

Definition 4. A goal $Q$ is narrowed into $Q^{\prime}$ at a position $\omega$ with the rule $l \rightarrow r$ and the substitution $\sigma$, if $\sigma$ is the most general unifier (mgu for short) of $l$ and

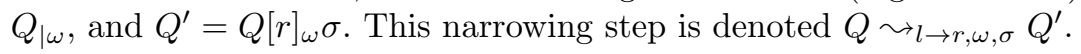

Indeed, every defined-innermost occurrence is narrowable:

Corollary 1. For any ground convergent rewrite system $\mathcal{R}$, for any equational goal $Q$, for any defined-innermost position $\omega \in \operatorname{Dom}(Q)$, for any ground substitution $\alpha$ and for any finite set $V$ of variables such that $\operatorname{Var}(Q) \cup \mathcal{D}$ om $(\alpha \downarrow) \subseteq V$, there exists a rule $l \rightarrow r \in \mathcal{R}$, a unifier $\sigma$ of $Q_{\mid \omega}$ and $l$, and a ground substitution $\mu$ such that $\sigma \mu_{\mid V}=(\alpha \downarrow)_{\mid V}$.

Proof. It is a consequence of the previous lemma and the classical narrowing lifting lemma $[18,20]$.

Thanks to these settings, we present in the next section, an induction based proof search system, relying on a main induction rule that uses narrowing to choose both the induction variables and the instantiation schema.

\section{A proof search system for induction}

The proof search system IndNarrow for inductive proofs introduced in this section is based on narrowing and rewriting. The main rule, called Induce, performs the induction step. This is the key point that provides a bridge between the implicit and explicit approaches of induction. Correctness and refutational completeness of this system are proved.

\subsection{The proof search system IndNarrow}

The rules in Figure 2 apply on sequents modulo of the form $\Gamma_{1} \mid \Gamma_{2} \vdash_{\mathcal{R E}_{1} \mid \mathcal{R E}_{2}} Q$, where $\Gamma_{1}$ is the deduction part of the definitions, $\mathcal{R} \mathcal{E}_{1}$ is their computational part; $\Gamma_{2}$ is the deduction part for other statements, $\mathcal{R} \mathcal{E}_{2}$ is their computational part; $Q$ is an equational goal. 


\begin{tabular}{|c|c|}
\hline Induce & $\begin{array}{l}\Gamma_{1} \mid \Gamma_{2} \vdash_{\mathcal{R E}_{1} \mid \mathcal{R E} \mathcal{E}_{2}} Q[t]_{\omega} \longmapsto \\
\bigodot_{\substack{\kappa \in \mathcal{R} \mathcal{E}_{1} \\
\sigma=m g u(t, l)}} \quad \Gamma_{1} \mid \Gamma_{2} \vdash_{\mathcal{R E}_{1} \mid \mathcal{R E}_{2} \sigma \cup \mathcal{R} \mathcal{E}_{\text {ind }}(Q,<) \sigma}\left(Q[r]_{\omega}\right) \sigma \\
\text { if } \kappa=l \rightarrow r \text { and } \omega \in D I(Q)\end{array}$ \\
\hline Orient & $\begin{array}{l}\Gamma_{1}\left|\Gamma_{2} \vdash_{\mathcal{R E}_{1} \cup\{\kappa\} \mid \mathcal{R} \mathcal{E}_{2}} Q \quad \longmapsto \Gamma_{1}\right| \Gamma_{2} \vdash_{\mathcal{R E}_{1} \cup\{l \rightarrow r\} \mid \mathcal{R} \mathcal{E}_{2}} Q \\
\text { if } \kappa=l \approx r \text { or } \kappa=r \approx l \text { and } l>r\end{array}$ \\
\hline Push $_{1}$ & $\Gamma_{1}, l \approx r\left|\Gamma_{2} \vdash_{\mathcal{R E}_{1} \mid \mathcal{R} \mathcal{E}_{2}} Q \quad \longmapsto \Gamma_{1}\right| \Gamma_{2} \vdash_{\mathcal{R E}_{1} \cup\{l \approx r\} \mid \mathcal{R} \mathcal{E}_{2}} Q$ \\
\hline $\mathbf{P u s h}_{2}$ & $\Gamma_{1}\left|\Gamma_{2}, l \approx r \vdash_{\mathcal{R E}_{1} \mid \mathcal{R E} \mathcal{E}_{2}} Q \quad \longmapsto \Gamma_{1}\right| \Gamma_{2} \vdash_{\mathcal{R E}_{1} \mid \mathcal{R} \mathcal{E}_{2} \cup\{l \approx r\}} Q$ \\
\hline Rewrite $_{1}$ & $\begin{array}{l}\Gamma_{1}\left|\Gamma_{2} \vdash_{\mathcal{R E}_{1} \cup\{\kappa\} \mid \mathcal{R E}_{2}} Q[l \sigma]_{\omega} \longmapsto \Gamma_{1}\right| \Gamma_{2} \vdash_{\mathcal{R E}_{1} \cup\{\kappa\} \mid \mathcal{R E}_{2}} Q[r \sigma]_{\omega} \\
\text { if } \kappa=l \rightarrow r \text { or } \kappa=l \approx r \text { or } \kappa=r \approx l\end{array}$ \\
\hline Rewrite $_{2}$ & $\begin{array}{l}\Gamma_{1}\left|\Gamma_{2} \vdash_{\mathcal{R E}_{1} \mid \mathcal{R E}_{2} \cup\{\kappa\}} Q[l \sigma]_{\omega} \longmapsto \Gamma_{1}\right| \Gamma_{2} \vdash_{\mathcal{R E}_{1} \mid \mathcal{R E}_{2} \cup\{\kappa\}} Q[r \sigma]_{\omega} \\
\text { if } \kappa=l \approx r \text { or } \kappa=r \approx l \text { or } \\
\kappa=\mathcal{R E}_{\text {ind }}(l \approx r) \mu \text { or } \kappa=\mathcal{R E}_{\text {ind }}(r \approx l) \mu \\
\text { and } \vec{x} \sigma<_{n} \vec{x} \mu \text { where } \vec{x}=\overrightarrow{\mathcal{V} a r(l \approx r)}\end{array}$ \\
\hline Trivial & $\Gamma_{1} \mid \Gamma_{2} \vdash_{\mathcal{R E}_{1} \mid \mathcal{R} \mathcal{E}_{2}} t \approx t \quad \longmapsto \diamond$ \\
\hline Refutation & $\begin{array}{ll}\Gamma_{1}\left|\Gamma_{2} \vdash_{\mathcal{R E}}\right| \mathcal{R E}_{2} Q & \longmapsto \text { Refutation } \\
\text { when no other rules can be applied }\end{array}$ \\
\hline
\end{tabular}

The distinction between $\Gamma_{1} / \mathcal{R} \mathcal{E}_{1}$ and $\Gamma_{2} / \mathcal{R E}_{2}$ is needed because in the Induce rule, only $\mathcal{R} \mathcal{E}_{1}$ is used for narrowing. For simplicity, we assume that $\mathcal{R} \mathcal{E}_{1}$ contains only unconditional rules or equalities and we assume from now on, that $\mathcal{R} \mathcal{E}_{1}$ is sufficiently complete.

$\Gamma_{2}$ is initialized with the proposition NI defined in subsection 1.2:

$$
N I: \quad \forall R \forall \tau \operatorname{Noeth}(R, \tau) \Rightarrow \forall P \operatorname{NoethInd}(P, R, \tau)
$$

and may contain other lemmas. $\mathcal{R} \mathcal{E}_{2}$ receives the induction hypotheses provided by some application of the rule Induce. So $\mathcal{R} \mathcal{E}_{2}$ may contain conditional equalities. $\odot$ operator that is an associative commutative operator on sequents with $\diamond$ as a neutral element.

The main rule is Induce as it performs the induction step. It uses narrowing to choose both the induction variable(s) and the instantiation schema. Narrowing is applied only at defined innermost positions (see Definition 3) $D I(Q)$ of the current goal $Q$. The other rules are doing the following: Trivial eliminates a trivial equation, Push pushes an equational hypothesis from the deduction part 
to the computational part, Orient orients an equation in the computational part into a rewrite rule, according to the term ordering, Rewrite (1 or 2) rewrites using a rule, an equation, or a smaller instance of a previous goal. Push and Rewrite are duplicated because of the $\Gamma_{1} / \mathcal{R} \mathcal{E}_{1}$ and $\Gamma_{2} / \mathcal{R} \mathcal{E}_{2}$ distinction.

\section{$3.2 \quad$ A simple example}

To get a better understanding of the way this set of rules is working, let us look at the proof of addition commutativity in Peano arithmetic. So, the goal is to prove:

$$
x+0 \approx x, x+s(y) \approx s(x+y) \mid N I \vdash_{\emptyset \mid \emptyset} X+Y \approx Y+X
$$

Applying $\mathbf{P u s h}_{1}$ twice, we get:

$$
\emptyset \mid N I \vdash_{x+0 \approx x, x+s(y) \approx s(x+y) \mid \emptyset} X+Y \approx Y+X
$$

Then, applying Orient twice gives us:

$$
\emptyset \mid N I \vdash_{x+0 \rightarrow x, x+s(y) \rightarrow s(x+y) \mid \emptyset} X+Y \approx Y+X
$$

We can now apply Induce since $\mathcal{R} \mathcal{E}_{1}=\{x+0 \rightarrow x, x+s(y) \rightarrow s(x+y)\}$ is confluent, terminating and sufficiently complete. This could be done at occurrence 1 or 2 of the goal. We arbitrary chose occurrence 1 and this leads us to prove the two sequents:

$$
\begin{aligned}
& \emptyset \mid N I \vdash_{\mathcal{R E}_{1} \mid \mathcal{R E}_{\text {ind }}\left(X+Y \approx Y+X,<, T_{\Sigma}^{2}\right)\left\{X \mapsto X_{1} ; Y \mapsto 0\right\}} X_{1} \approx 0+X_{1} \\
& \emptyset \mid N I \vdash_{\mathcal{R E}_{1} \mid \mathcal{R E}_{\text {ind }}\left(X+Y \approx Y+X,<, T_{\Sigma}^{2}\right)\left\{X \mapsto X_{1} ; Y \mapsto s\left(Y_{1}\right)\right\}} s\left(X_{1}+Y_{1}\right) \approx s\left(Y_{1}\right)+X_{1}
\end{aligned}
$$

We have now to prove in particular that 0 is left-neutral. The only applicable rule on that goal is Induce again and we get the two new subgoals:

$$
\begin{aligned}
& \emptyset \mid N I \vdash^{\mathcal{R} \mathcal{E}_{1} \mid} \mathcal{R E}_{\text {ind }}\left(X+Y \approx Y+X,<, T_{\sum}^{2}\right)\{X \mapsto 0 ; Y \mapsto 0\} \\
& \mathcal{R E}_{\text {ind }}\left(X_{1} \approx 0+X_{1},<, T_{\Sigma}\right)\left\{X_{1} \mapsto 0\right\}
\end{aligned}
$$

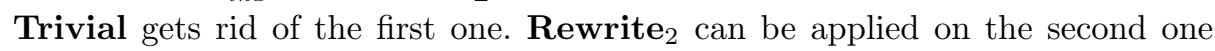
since, because of narrowing, the goal has been reduced and therefore the induction hypothesis can now be used. We get:

$$
\emptyset \mid N I \vdash_{\mathcal{R E}_{1} \mid \mathcal{R E}_{\text {ind }}\left(X+Y \approx Y+X,<, T_{\sum}^{2}\right)\left\{X \mapsto s\left(X_{2}\right) ; Y \mapsto 0\right\}} s\left(X_{2}\right) \approx s\left(X_{2}+0\right)
$$

Applying now Rewrite R $_{1}$ proves that 0 is left-neutral for addition. We are left with the goal $s\left(X_{1}+Y_{1}\right) \approx s\left(Y_{1}\right)+X_{1}$ and we will make precise later on how the proof search finishes.

\subsection{Soundness of IndNarrow}

Soundness amounts to show that for each rule of the proof search system IndNarrow of the form:

$$
\Gamma_{1}\left|\Gamma_{2} \vdash_{\mathcal{R E}_{1} \mid \mathcal{R} \mathcal{E}_{2}} Q \longmapsto \bigodot_{i \in I} \Gamma_{1}^{i}\right| \Gamma_{2}^{i} \vdash_{\mathcal{R E}_{1}^{i} \mid \mathcal{R} \mathcal{E}_{2}^{i}} Q^{i}
$$

then $\Gamma_{1} \mid \Gamma_{2}, \vec{x} \in \mathcal{T}(\Sigma)^{n} \vdash_{\mathcal{R E}_{1} \mid \mathcal{R} \mathcal{E}_{2}} Q$ is derivable provided all the $\Gamma_{1}^{i} \mid \Gamma_{2}^{i}, \overrightarrow{x^{i}} \in$ $\mathcal{T}(\Sigma)^{n^{i}} \vdash_{\mathcal{R E}_{1}^{i} \mid \mathcal{R} \mathcal{E}_{2}^{i}} Q^{i}$ are. In what follows, we assume that all variables in $\Gamma$ are universally quantified. 
Let us first state a few basic rules which are needed in the soundness proof.

Lemma 4. The following rules (where $P, P_{1}, P_{2}$ are propositions) are derivable in the sequent calculus modulo:

1.

$$
\frac{\Gamma \vdash_{\mathcal{R E}} P_{1} \Rightarrow P_{2}, \Delta}{\Gamma, P_{1} \vdash_{\mathcal{R E}} P_{2}, \Delta} i m p
$$

2 .

$$
\overline{\Gamma, x=y \vdash x \approx y} r e f
$$

3.

$$
\frac{\Gamma \vdash_{\mathcal{R E}} \forall x \alpha(x) \approx \beta(x) \quad \Gamma \vdash_{\mathcal{R E} \alpha} P \alpha}{\Gamma \vdash_{\mathcal{R E} \beta} P \beta} r_{e}
$$

4.

$$
\frac{\Gamma, \vec{x} \in \mathcal{T}(\Sigma)^{n} \vdash_{\mathcal{R E}} P, \Delta}{\Gamma \vdash_{\mathcal{R E} \alpha} P \alpha, \Delta} r_{\alpha} \quad \text { if }\left\{\begin{array}{l}
\alpha \in S u b s t^{\Sigma} \\
\vec{x} \text { is the vector of free variables of } \mathcal{R E} \cup P
\end{array}\right.
$$

5.

$$
\frac{\bigwedge_{\alpha \in \text { Subst }^{\Sigma}} \Gamma \vdash_{\mathcal{R E} \alpha} P \alpha}{\Gamma, \vec{x} \in \mathcal{T}(\Sigma)^{n} \vdash_{\mathcal{R E}} P} r_{\vec{x}} \quad \text { if } \vec{x} \text { is the vector of free variables of } \mathcal{R E} \cup P
$$

6. For any proposition $P$ and for any integer $n$, if $|\mathcal{V} \operatorname{ar}(P) \cup \mathcal{V} \operatorname{ar}(\mathcal{R E})|=n$, if the proposition $P$ is inductive in some context $\Gamma \cup \mathcal{R E}$ with respect to the order $<_{n}$, and if this order is Noetherian in this context, then the proposition $P$ is valid in the context $\Gamma \cup \mathcal{R E}$, whenever it contains the proposition $N I=$ $\forall R \forall \tau(\operatorname{Noeth}(R, \tau) \Rightarrow \forall P$ NoethInd $(P, R, \tau))$ (see subsection 3.1)

7.

$$
\frac{\Gamma, \vec{x} \in \mathcal{T}(\Sigma)^{n} \vdash_{\mathcal{R E}_{\mathcal{E}} \mathcal{R E}_{\text {ind }}(P,<)} P \quad \Gamma \vdash_{\mathcal{R E}} \operatorname{Noeth}\left(<_{n}, \mathcal{T}(\Sigma)^{n}\right)}{\Gamma, \vec{x} \in \mathcal{T}(\Sigma)^{n} \vdash_{\mathcal{R E}} P} r_{I}
$$

if $\vec{x}$ is the vector of free variables of $\mathcal{R E} \cup P$

We are now ready to prove soundness of IndNarrow in the sequent calculus modulo by considering in turn each inference rule of IndNarrow.

Theorem 2. For any contexts $\Gamma_{1}, \Gamma_{2}$, rewrite systems $\mathcal{R E}_{1}, \mathcal{R E}_{2}$, equational goal $Q$, occurrence $\omega \in D I(Q)$ and integer $n$, let us assume that:

1. Induce is applied on to get

$$
\Gamma_{1}\left|\Gamma_{2} \vdash \mathcal{R E}_{1}\right| \mathcal{R} \mathcal{E}_{2} Q[t]_{\omega}
$$

$\bigodot_{\substack{l \rightarrow r \in \mathcal{R} \mathcal{E}_{1} \\ \sigma=m g u(t, l)}} \Gamma_{1} \mid \Gamma_{2} \vdash_{\mathcal{R E}_{1} \mid \mathcal{R E}_{2} \sigma \cup \mathcal{R} \mathcal{E}_{\text {ind }}(Q,<) \sigma}\left(Q[r]_{\omega}\right) \sigma ;$

2. $\mathcal{R E}_{1}$ is ground convergent and sufficiently complete; 
3. $<$ is Noetherian, so that $\Gamma_{1} \cup \Gamma_{2} \vdash_{\mathcal{R} \mathcal{E}_{1} \cup \mathcal{R} \mathcal{E}_{2}} \operatorname{Noeth}\left(<_{n}, \mathcal{T}(\Sigma)^{n}\right)$;

4. for any rewrite rule $l \rightarrow r \in \mathcal{R E}_{1}$, when $\sigma=m g u(t, l)$ and $\vec{x}_{\sigma} \in \mathcal{X}^{n_{\sigma}}$ is the vector of free variables of $\mathcal{R} \mathcal{E} \sigma \cup Q \sigma$, the sequent

$\Gamma_{1} \cup \Gamma_{2}, \vec{x}_{\sigma} \in \mathcal{T}(\Sigma)^{n_{\sigma}} \vdash_{\mathcal{R E}_{1} \cup \mathcal{R} \mathcal{E}_{2} \sigma \cup\left\{\mathcal{R E}_{\text {ind }}(Q,<) \sigma\right\}}\left(Q[r]_{\omega}\right) \sigma$ is derivable in the sequent calculus modulo.

Then, the sequent

$\Gamma_{1} \cup \Gamma_{2}, \vec{x} \in \mathcal{T}(\Sigma)^{n} \vdash_{\mathcal{R} \mathcal{E}_{1} \cup \mathcal{R} \mathcal{E}_{2}} Q[t]_{\omega}$ is derivable in the sequent calculus modulo.

Proof. First, let us introduce the following notations:

$$
\begin{array}{lll}
\Gamma & \text { denotes } & \Gamma_{1} \cup \Gamma_{2} \\
\mathcal{R E} & \text { denotes } & \mathcal{R} \mathcal{E}_{1} \cup \mathcal{R} \mathcal{E}_{2} \\
\mathcal{R} \mathcal{E}^{\prime} & \text { denotes } & \mathcal{R E} \cup\left\{\mathcal{R E}_{\text {ind }}(Q,<)\right\} \\
\mathcal{R} \mathcal{E} \sigma & \text { denotes } & \mathcal{R} \mathcal{E}_{1} \cup \mathcal{R} \mathcal{E}_{2} \sigma \\
\mathcal{R} \mathcal{E}^{\prime} \sigma & \text { denotes } & \mathcal{R} \mathcal{E} \sigma \cup\left\{\mathcal{R E}_{\text {ind }}(Q,<)\right\} \sigma
\end{array}
$$

Let $V$ denote a finite set of variables and $\alpha$ a ground substitution, such that

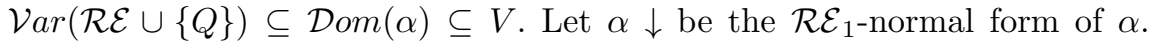
According to the narrowing lemma, we have:

$$
\sigma \mu_{\mid V}=(\alpha \downarrow)_{\mid V}
$$

for some substitution $\mu$. Let us consider the following derivations.

$\Pi_{1}$

$$
\overline{\forall x x(\alpha \downarrow)=x \sigma \mu \vdash_{\mathcal{R E}} \forall x x \alpha=x \sigma \mu} A x
$$

$\Pi_{2}$

$$
\frac{\vdash \forall x x(\alpha \downarrow)=x \sigma \mu \quad(\text { by } 5)}{\vdash_{\mathcal{R E}} \forall x x(\alpha \downarrow)=x \sigma \mu, \forall x x \alpha=x \sigma \mu} w+p u s h
$$

$\Pi_{3}$

$$
\begin{aligned}
& \frac{\Pi_{1} \Pi_{2}}{\vdash_{\mathcal{R E}} \forall x x \alpha=x \sigma \mu} \text { cut }
\end{aligned}
$$

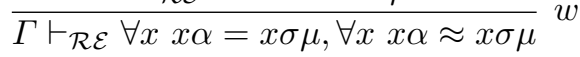

$\Pi_{4}$ :

$$
\begin{aligned}
& \overline{\Gamma, x \alpha=x \sigma \mu \vdash x \alpha \approx x \sigma \mu} \text { ref } \\
& \frac{\Gamma, x \alpha=x \alpha=x \sigma \mu \vdash x \alpha \approx x \sigma \mu}{\Gamma, \forall x x \alpha=l} \\
& \frac{\overline{\Gamma, \forall x x \alpha=x \sigma \mu \vdash \forall x x \alpha \approx x \sigma \mu}}{\Gamma, \forall x x \alpha=x \sigma \mu \vdash_{\mathcal{R E}} \forall x x \alpha \approx x \sigma \mu} w+p u s h
\end{aligned}
$$

$\Pi_{5}$ :

$$
\frac{\Pi_{3} \Pi_{4}}{\Gamma \vdash_{\mathcal{R E}} \forall x x \alpha \approx x \sigma \mu} c u t
$$


$\Pi_{6}:$

$$
\frac{\Gamma, \vec{x}_{\sigma} \in \mathcal{T}(\Sigma)^{n} \vdash_{\mathcal{R E}^{\prime} \sigma} Q \sigma[r \sigma]_{\mid \omega}}{\Gamma, \vec{x}_{\sigma} \in \mathcal{T}(\Sigma)^{n} \vdash_{\mathcal{R E}^{\prime} \sigma} Q \sigma, Q \sigma[r \sigma]_{\mid \omega}} w
$$

$\Pi_{7}$

$$
\frac{\overline{Q \sigma[r \sigma]_{\mid \omega} \vdash_{\mathcal{R E}_{1}} Q \sigma[l \sigma]_{\mid \omega}} A x}{\frac{\Gamma_{, \vec{x}_{\sigma} \in \mathcal{T}(\Sigma)^{n}, Q \sigma[r \sigma]_{\mid \omega} \vdash_{\mathcal{R E}^{\prime} \sigma} Q \sigma[l \sigma]_{\mid \omega}}}{\Gamma, \vec{x}_{\sigma} \in \mathcal{T}(\Sigma)^{n}, Q \sigma[r \sigma]_{\mid \omega} \vdash_{\mathcal{R E}^{\prime} \sigma} Q \sigma}} w
$$

(since $l \sigma=t \sigma$ and $Q=Q[t]_{\mid \omega}$ )

$\Pi_{1, \sigma}$ :

$$
\frac{\Pi_{6} \Pi_{7}}{\Gamma, \vec{x}_{\sigma} \in \mathcal{T}(\Sigma)^{n} \vdash_{\mathcal{R E}^{\prime} \sigma} Q \sigma} \text { cut }
$$

Denoting $\mathcal{P E}_{\text {ind }}(Q)$ the canonical proposition associated to $\mathcal{R E}_{\text {ind }}(Q,<)$, this leads to:

$\Pi_{2, \sigma}$

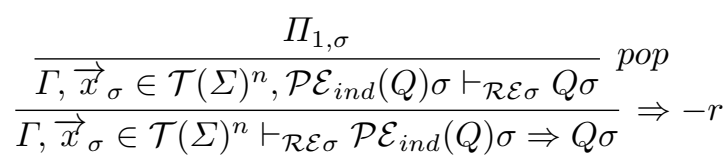

Since the proposition $\mathcal{P} \mathcal{E}_{\text {ind }}(Q) \sigma \Rightarrow{ }_{\mathcal{R E}} Q \sigma$ is equivalent to $\left(\mathcal{P E}_{\text {ind }}(Q) \Rightarrow_{\mathcal{R E}} Q\right) \sigma$, we have:

$\Pi_{\sigma, \mu}$ :

$$
\frac{\Pi_{2, \sigma}}{\Gamma \vdash_{\mathcal{R E} \sigma \mu}\left(\mathcal{P E}_{\text {ind }}(Q) \Rightarrow Q\right) \sigma \mu} r_{\mu}
$$

$\Pi_{\alpha}:$

$$
\frac{\Pi_{\sigma, \mu} \Pi_{5}}{\Gamma \vdash_{\mathcal{R E} \alpha}\left(\mathcal{P \mathcal { E } _ { \text { ind } } ( Q ) \Rightarrow Q ) \alpha}\right.} r_{e}
$$

And since $\alpha$ is any ground substitution, we have:

$\Pi_{\vec{x}}$ :

$$
\frac{\bigwedge_{\alpha \in \text { Subst }^{\Sigma}} \Pi_{\alpha}}{\frac{\Gamma_{\Gamma, \vec{x} \in \mathcal{T}(\Sigma)^{n} \vdash_{\mathcal{R E}} \mathcal{P} \mathcal{E}_{\text {ind }}(Q) \Rightarrow Q}^{\Gamma, \vec{x} \in \mathcal{T}(\Sigma)^{n}, \mathcal{P} \mathcal{E}_{\text {ind }}(Q) \vdash_{\mathcal{R E}} Q}}{\Gamma, \vec{x} \in \mathcal{T}(\Sigma)^{n} \vdash_{\mathcal{R E} \cup \mathcal{R} \mathcal{E}_{\text {ind }}(Q,<)} Q} r_{\vec{x}}}{ }^{i m p}
$$

$\Pi:$

$$
\frac{\Pi_{\vec{x}} \quad \Gamma \vdash_{\mathcal{R E}} \operatorname{Noeth}\left(<_{n}, \mathcal{T}(\Sigma)^{n}\right)}{\Gamma, \vec{x} \in \mathcal{T}(\Sigma)^{n} \vdash_{\mathcal{R E}} Q} r_{I}
$$

and we are done.

Soundness of Push is simply a consequence of soundness of the sequent calculus modulo.

Let us now look at the Rewrite inferences. 
Theorem 3. For all contexts $\Gamma_{1}, \Gamma_{2}$, for all rewrite systems $\mathcal{R E}_{1}, \mathcal{R E}_{2}$, for any equational goal $Q$, let us assume that:

1. Rewrite $\Gamma_{1,2}$ is applied on to get:

$$
\Gamma_{1} \mid \Gamma_{2} \vdash_{\mathcal{R} \mathcal{E}_{1} \mid \mathcal{R} \mathcal{E}_{2}} Q
$$

$\Gamma_{1} \mid \Gamma_{2} \vdash{ }_{\mathcal{R E}_{1} \mid \mathcal{R E}_{2}} Q^{\prime}$

2. The sequent $\Gamma_{1} \mid \Gamma_{2} \vdash_{\mathcal{R E}_{1} \mid \mathcal{R} \mathcal{E}_{2}} Q^{\prime}$ admits a proof.

Then, the sequent $\Gamma_{1} \mid \Gamma_{2} \vdash_{\mathcal{R} \mathcal{E}_{1} \mid \mathcal{R} \mathcal{E}_{2}} Q$ is derivable in the sequent calculus modulo.

Proof. Let us use the same notations as in the previous theorem and detail the most elaborated case, namely the application of Rewrite $_{2}$. By assumption 1 , and by definition of this rule, there exist

$\kappa \in \mathcal{R E},(l, r) \in \mathcal{T}(\Sigma, \mathcal{X})^{2}, \omega \in \mathcal{D o m}(Q)$, and $\sigma \in \operatorname{Subst}^{\mathcal{T}(\Sigma, \mathcal{X})}$, such that:

$-\kappa=l \approx r$ or $\kappa=r \approx l$ or

$\exists \mu\left(\mu \in \operatorname{Subst}^{\mathcal{T}(\Sigma, \mathcal{X})}\right)$ and $\left(\kappa=\mathcal{R E}_{\text {ind }}(l \approx r) \mu\right.$ or $\left.\kappa=\mathcal{R E}_{\text {ind }}(r \approx l) \mu\right)$

$-\vec{y} \in \mathcal{X}^{m}$ such that $\vec{y}=\overrightarrow{\mathcal{V} a r(l \approx r)}$ and $\vec{y} \sigma<_{m} \vec{y} \mu$

- $Q=Q[l \sigma]_{\mid \omega}$ and $Q^{\prime}=Q[r \sigma]_{\mid \omega}$.

Let us then consider the following proof:

$\Pi_{1}$ :

$$
\frac{\Gamma \vdash_{\mathcal{R E}} \vec{y} \sigma<_{m} \vec{y} \mu}{\Gamma, Q[r \sigma] \vdash_{\mathcal{R E}} \vec{y} \sigma<_{m} \vec{y} \mu, Q[l \sigma]} w
$$

$\Pi_{2}$ :

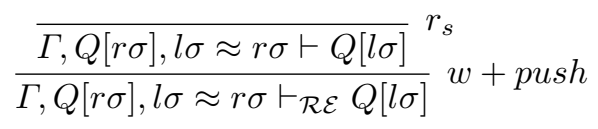

$\Pi_{3}:$

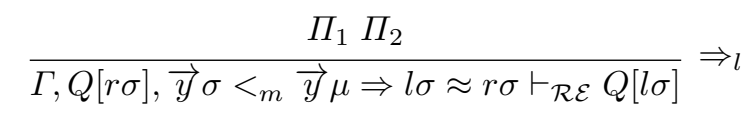

$\Pi_{4}:$

$$
\frac{\frac{\Pi_{3}}{\Gamma, Q[r \sigma], \forall \underline{y} \vec{y}<_{m} \vec{y} \mu \Rightarrow(l \approx r)\{\vec{y} / \vec{y}\} \vdash_{\mathcal{R E}} Q[l \sigma]}}{\Gamma, Q[r \sigma] \vdash_{\mathcal{R E} \cup\{\kappa\}} Q[l \sigma]} \forall_{l} \text { push }
$$

Now, since $\kappa \in \mathcal{R E}$, we have:

$\Pi_{4}$ :

$$
\frac{\frac{\Pi_{3}}{\Gamma, Q[r \sigma], \forall \underline{y} \vec{y}<_{m} \vec{y} \mu \Rightarrow(l \approx r)\{\vec{y} / \vec{y}\} \vdash_{\mathcal{R E}} Q[l \sigma]}}{\Gamma, Q[r \sigma] \vdash_{\mathcal{R E}} Q[l \sigma]} \forall_{l} \text { push }
$$

$\Pi_{5}:$

$$
\frac{\Pi_{4}}{\Gamma, \vec{x} \in \mathcal{T}(\Sigma)^{n}, Q[r \sigma] \vdash_{\mathcal{R E}} Q[l \sigma]} w
$$


$\Pi_{6}:$

$$
\frac{\Gamma, \vec{x} \in \mathcal{T}(\Sigma)^{n} \vdash_{\mathcal{R E}} Q[r \sigma] \text { (assumed) }}{\Gamma, \vec{x} \in \mathcal{T}(\Sigma)^{n} \vdash_{\mathcal{R E}} Q[r \sigma], Q[l \sigma]} w
$$

$\Pi:$

$$
\frac{\Pi_{5} \Pi_{6}}{\Gamma, \vec{x} \in \mathcal{T}(\Sigma)^{n} \vdash_{\mathcal{R E}} Q[l \sigma]} \text { cut }
$$

and we are done.

We have already proved soundness of the rewrite system IndNarrow \ \{Orient\}. Now, it is easy to see that, for all contexts $\Gamma, \Gamma^{\prime}$, for all rewrite systems $\mathcal{R E}, \mathcal{R \mathcal { E } ^ { \prime }}$, and for all equational goals $Q, Q^{\prime}$, one can build a derivation $\Gamma \vdash_{\mathcal{R E}} Q \stackrel{*}{{ }^{\prime}}$ IndNarrow $\backslash\{$ Orient $\} \Gamma^{\prime} \vdash_{\mathcal{R E}} Q^{\prime}$ whenever there exists a derivation $\Gamma \vdash_{\mathcal{R E}} Q \stackrel{*}{\mapsto}$ IndNarrow $\Gamma^{\prime} \vdash_{\mathcal{R E}} Q^{\prime}$. Therefore, soundness of IndNarrow is a consequence of soundness of IndNarrow $\backslash\{$ Orient $\}$.

\subsection{Example (continued)}

Remember that we need to prove:

$$
\emptyset \mid N I \vdash_{\mathcal{R E}_{1} \mid \mathcal{R} \mathcal{E}_{\text {ind }}\left(X+Y \approx Y+X,<, T_{\Sigma}^{2}\right)\left\{X \mapsto X_{1} ; Y \mapsto s\left(Y_{1}\right)\right\}} s\left(X_{1}+Y_{1}\right) \approx s\left(Y_{1}\right)+X_{1}
$$

We can apply Induce at position 2 , leading to:

$$
\begin{aligned}
& \emptyset \mid N I \vdash_{\mathcal{R E}_{1} \mid \mathcal{R E}_{2}^{\prime}} s\left(0+Y_{3}\right) \approx s\left(Y_{3}\right) \\
& \emptyset \mid N I \vdash_{\mathcal{R E}_{1} \mid \mathcal{R E}_{\text {ind }}\left(X+Y \approx Y+X,<, T_{\Sigma}^{2}\right) \sigma_{1} \sigma_{2}} s\left(s\left(X_{3}\right)+Y_{3}\right) \approx s\left(s\left(Y_{3}\right)+X_{3}\right) \\
& \mathcal{R E}_{\text {ind }}\left(s\left(X_{1}+Y_{1}\right) \approx s\left(Y_{1}\right)+X_{1},<, T_{\Sigma}^{2}\right) \sigma_{2}
\end{aligned}
$$

where $\mathcal{R} \mathcal{E}_{2}^{\prime}$ is easy to explicit and where $\sigma_{1}=\left\{X \mapsto X_{1} ; Y \mapsto s\left(Y_{1}\right)\right\}$ (coming from the previous application of Induce) and $\sigma_{2}=\left\{X_{1} \mapsto s\left(X_{3}\right) ; Y_{1} \mapsto Y_{3}\right\}$. In the same way as before, the goal $s\left(0+Y_{3}\right) \approx s\left(Y_{3}\right)$ is solved. Reducing with the Rewrite rules and using Theorem 1 to check the conditions leads directly to the proof of the last goal, therefore finishing the proof.

Notice that, following the soundness proof above, the proof search developed in the example can be straightforwardly expanded into a sequent calculus proof.

\subsection{Refutational correctness}

Refutational correctness amounts to show that for each rule of the proof search system IndNarrow of the form:

$$
\Gamma_{1}\left|\Gamma_{2} \vdash_{\mathcal{R E}_{1} \mid \mathcal{R E}_{2}} Q \longmapsto \bigodot_{i \in I} \Gamma_{1}^{i}\right| \Gamma_{2}^{i} \vdash_{\mathcal{R E}_{1}^{i} \mid \mathcal{R} \mathcal{E}_{2}^{i}} Q^{i}
$$

then all the $\Gamma_{1}^{i} \mid \Gamma_{2}^{i}, \overrightarrow{x^{i}} \in \mathcal{T}(\Sigma)^{n^{i}} \vdash_{\mathcal{R E} \mathcal{E}_{1}^{i} \mid \mathcal{R E} \mathcal{E}_{2}^{i}} Q^{i}$ are derivable provided $\Gamma_{1} \mid \Gamma_{2}, \vec{x} \in$ $\mathcal{T}(\Sigma)^{n} \vdash_{\mathcal{R E}_{1} \mid \mathcal{R E}_{2}} Q$ is.

We detail here the most delicate point which is again the case of the rule Induce, addressed in the following theorem. 
Theorem 4. For all contexts $\Gamma_{1}, \Gamma_{2}$, for all rewrite systems $\mathcal{R E}_{1}, \mathcal{R E}_{2}$, for any equational goal $Q$, for any $\omega \in D I(Q)$, and for any integer $n$,

If

$$
\Gamma_{1}\left|\Gamma_{2} \vdash_{\mathcal{R E}_{1} \mid \mathcal{R E}_{2}} Q[t]_{\omega} \stackrel{\text { Induce }}{\longmapsto} \bigodot_{\substack{l \rightarrow r \in \mathcal{R} \mathcal{E}_{1} \\ \sigma=m g u(t, l)}} \Gamma_{1}\right| \Gamma_{2} \vdash_{\mathcal{R E}_{1} \mid \mathcal{R E}_{2} \sigma \cup \mathcal{R} \mathcal{E}_{\text {ind }}(Q,<) \sigma} Q[r]_{w} \sigma
$$

and if the sequent $\Gamma_{1} \cup \Gamma_{2}, \vec{x} \in \mathcal{T}(\Sigma)^{n} \vdash_{\mathcal{R E}_{1} \cup \mathcal{R} \mathcal{E}_{2}} Q[t]_{\omega}$ (where $\vec{x} \in \mathcal{X}^{n}$ denotes the vector of free variables of $\mathcal{R} \mathcal{E}_{2} \cup Q$ ) admits a proof in sequent calculus modulo, then, for any $\sigma=m g u(t, l)$, for any integer $n_{\sigma}$, for any vector of free variables $\vec{x}_{\sigma}$ of $\mathcal{R} \mathcal{E} \sigma \cup Q \sigma$ in $\mathcal{X}^{n_{\sigma}}$, one can build a proof of

$$
\Gamma_{1} \cup \Gamma_{2}, \vec{x}_{\sigma} \in \mathcal{T}(\Sigma)^{n_{\sigma}} \vdash_{\mathcal{R E}_{1} \cup \mathcal{R} \mathcal{E}_{2} \sigma \cup\left\{\mathcal{R E}_{\text {ind }}(Q,<) \sigma\right\}} Q[r]_{\omega} \sigma
$$

Proof. Recall the notations 4 . Let $\sigma=m g u(t, l)$. For any ground substitution $\mu$, we have:

$\Pi_{\sigma, \mu}$

$$
\frac{\Gamma, \vec{x} \in \mathcal{T}(\Sigma)^{n} \vdash_{\mathcal{R E}} Q}{\Gamma \vdash_{\mathcal{R E} \sigma \mu} Q \sigma \mu} r_{\sigma \mu}
$$

Now, let us consider the following derivations:

$$
\Pi_{1, \sigma}:
$$

$$
\frac{\bigwedge_{\mu \in S u b s t^{\Sigma}} \Pi_{\sigma, \mu}}{\Gamma, \vec{x}_{\sigma} \in \mathcal{T}(\Sigma)^{n_{\sigma}} \vdash_{\mathcal{R E} \sigma} Q \sigma} r_{\vec{x}_{\sigma}}
$$

$\Pi_{2, \sigma}$ :

$$
\frac{\Pi_{1, \sigma}}{\Gamma, \vec{x}_{\sigma} \in \mathcal{T}(\Sigma)^{n_{\sigma}} \vdash_{\mathcal{R E} \sigma} Q \sigma, Q \sigma[r \sigma]_{\mid \omega}} w
$$

Denoting $T h_{\mathcal{R E}_{2} \sigma}$ the canonical theory associated to $\mathcal{R} \mathcal{E}_{2} \sigma$, and since $\mathcal{R E} \sigma=\mathcal{R E}_{1} \cup \mathcal{R} \mathcal{E}_{2} \sigma$, we obtain:

$$
\begin{aligned}
& \Pi_{3, \sigma}:
\end{aligned}
$$

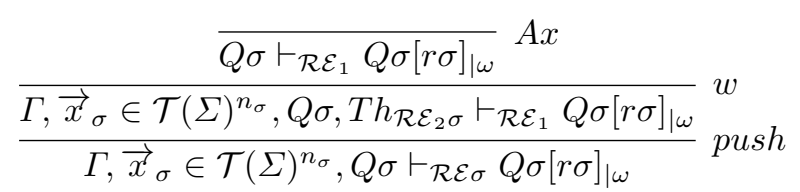

Denoting $\mathcal{P} \mathcal{E}_{\text {ind }}(Q)$ the canonical proposition associated to $\mathcal{R E}_{\text {ind }}(Q,<)$, this leads to: 
$\Pi_{4, \sigma}:$

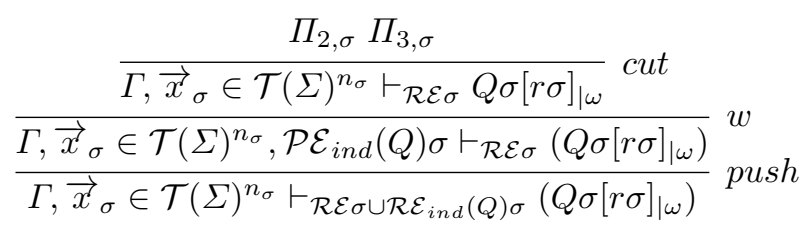

and we are done.

As a corollary of Theorem 4, we get:

Theorem 5. The proof search system IndNarrow is refutationally correct.

Proof. Induce being handled in Theorem 4, the other inference rules Rewrite and Orient are proved refutationally correct, in similar ways. Correctness of the other rules is a consequence of correctness of deduction modulo.

Moreover, thanks to the Refutation rule which applies when no other rule of IndNarrow can be applied, we can also prove that when a derivation ends with Refutation, the original sequent has no proof in deduction modulo.

Lemma 5. For all contexts $\Gamma_{1}, \Gamma_{2}$, for all rewrite systems $\mathcal{R E}_{1}, \mathcal{R} \mathcal{E}_{2}$, if:

$$
\Gamma_{1} \mid \Gamma_{2} \vdash_{\mathcal{R E}_{1} \mid \mathcal{R E}_{2}} Q \longmapsto \text { Refutation }
$$

then, the sequent $\Gamma_{1} \mid \Gamma_{2} \vdash_{\mathcal{R E}_{1} \mid \mathcal{R E}_{2}} Q$ has no proof.

Proof. If $Q$ contains a defined symbol, there exists a defined-innermost position in $\operatorname{Dom}(Q)$, therefore one can apply the rule Induce, and there is a contradiction. Since the rule Trivial cannot be applied either, we have $Q=t \approx t^{\prime}$, with $t, t^{\prime}$ constructor terms that are not syntactically equal. Therefore, the sequent $\Gamma_{1} \mid \Gamma_{2} \vdash_{\mathcal{R E}_{1} \mid \mathcal{R} \mathcal{E}_{2}} Q$ has no proof, since the constructors are assumed to be free and $\approx$ satisfies the axioms of equality.

Theorem 6. For all contexts $\Gamma_{1}, \Gamma_{2}$, for all rewrite systems $\mathcal{R} \mathcal{E}_{1}, \mathcal{R} \mathcal{E}_{2}$, if there exists an IndNarrow-derivation

$$
\Gamma_{1} \mid \Gamma_{2} \vdash_{\mathcal{R E}_{1} \mid \mathcal{R} \mathcal{E}_{2}} Q \longmapsto \stackrel{*}{\longmapsto}{ }_{\text {IndNarrow }} \text { Refutation }
$$

then, the sequent $\Gamma_{1} \mid \Gamma_{2} \vdash_{\mathcal{R E}_{1} \mid \mathcal{R} \mathcal{E}_{2}} Q$ has no proof.

Proof. Assume: $\Gamma_{1} \mid \Gamma_{2} \vdash_{\mathcal{R E}_{1} \mid \mathcal{R} \mathcal{E}_{2}} Q \longmapsto_{\text {IndNarrow }}^{*}$ Refutation

There exist contexts $\Gamma_{1}^{\prime}, \Gamma_{2}^{\prime}$, rewrite systems $\mathcal{R} \mathcal{E}_{1}^{\prime}, \mathcal{R} \mathcal{E}_{2}^{\prime}$ and an equational goal $Q^{\prime}$ such that:

$$
\Gamma_{1}\left|\Gamma_{2} \vdash_{\mathcal{R E} \mathcal{E}_{1} \mid \mathcal{R} \mathcal{E}_{2}} Q \longmapsto \stackrel{*}{*}{ }_{\text {IndNarrow }} \Gamma_{1}^{\prime}\right| \Gamma_{2}^{\prime} \vdash_{\mathcal{R E}_{1}^{\prime} \mid \mathcal{R E} \mathcal{E}_{2}^{\prime}} Q^{\prime} \longmapsto_{\text {IndNarrow }} \text { Refutation }
$$

And, by lemma 5 , the sequent $\Gamma_{1}^{\prime}\left|\Gamma_{2}^{\prime} \vdash_{\mathcal{R E}}^{\prime}\right| \mathcal{R \mathcal { E } _ { 2 } ^ { \prime }} Q^{\prime}$ has no proof. Therefore, by the refutation correctness of IndNarrow (Theorem 5), $\Gamma_{1} \mid \Gamma_{2} \vdash_{\mathcal{R E}_{1} \mid \mathcal{R E}_{2}} Q$ has no proof either. 
Refutational completeness of the proof search system IndNarrow, i.e. proving that if a sequent has no proof in deduction modulo, then there is a derivation leading to Refutation, is not detailed here, but this result can be derived from the proof given in [24] in the more complex context where associative commutative theories are considered.

\section{Conclusion}

We have shown how narrowing can provide the inference mechanism to perform inductive proof search. Instead of pre-computing induction schemata and induction variables, this approach has the advantage to target exactly which variables should be instantiated and how. Moreover, because the method derives directly from the deduction modulo framework, we take benefit from a direct translation from a successful proof search derivation to a sequent calculus modulo proof. Last but not least, the fact that we are precisely specifying the conditions on the induction ordering allows us to narrow the search space. Although heuristics for lemma speculation, generalisation and induction rule choice are always in need for improvement in inductive proof search, it was not the aim of our work here. For instance, a suitable noetherian ordering is implicitly assumed throughout the paper, rather than discovered by the search strategy like in explicit induction methods. Finally, it is now possible to have an automated construction of inductive proofs into the sequent calculus for insertion into proof assistants.

At the proof level, the general framework of deduction modulo is quite relevant to keep at the deduction level only the true deduction steps like modus ponens and to delegate all computational steps on propositions or terms to specialized provers using equational and rewriting techniques. Then, some parts of the proofs can be deferred to aside computations, while the true skeleton of the proof is being built. At the checking level, the experiences described in [9] of translating equational and inductive proofs to proof terms for Coq should be quite useful.

If the approach is theoretically fruitful and enlightens the relationship between rewrite based induction methods and Noetherian induction, we are clearly in need of an implementation of the results presented here. Our goal will be to achieve this as a way to mechanize proof search in a proof assistant based on type theory and the rewriting calculus $[2,26]$. Along these lines, a first prototype has been written in collaboration with Paul Brauner and is described in [24]. Moreover our approach provides the ability to use an induction principle based on Noetherian rewrite systems, therefore strongly enhancing over the structural induction principle which is, in practice, used in most of the current proof assistants.

This narrowing based approach opens also new fundamental questions, let us mention three of them. The first one concerns its relationship with the very useful rippling [5] technique. Indeed, in a way related to rippling, narrowing makes explicit and links with a Noetherian rewrite system what we are in need for inductively proving a goal. This analogy should be deepened and possibly 
exploited. A second one concerns the extension of rewrite based inductive theorem proving to class rewriting. This has been explored in particular in [3] for associative-commutative theories. The genericity of narrowing modulo may enlighten and ease the use of such class rewrite systems to base inductive proof search. This has been explored in [24]. The third one concerns inductive proof by consistency which is indeed at the source of the use of rewrite techniques for induction $[22,15,6,25]$. The relationship between deduction modulo and such a consistency technique is worth to be better understood.

Acknowledgments: Many thanks to the members of the Protheo team in which, from 2004 to 2007, the results presented in this paper have been elaborated, benefitting of stimulating discussions on many of the subjects developed in this paper. Special thanks to Eric Deplagne whose PhD initiated the ideas on which this paper is based.

\section{References}

1. F. Baader and T. Nipkow. Term Rewriting and all That. Cambridge University Press, 1998.

2. G. Barthe, H. Cirstea, C. Kirchner, and L. Liquori. Pure Patterns Type Systems. In Principles of Programming Languages - POPL2003, New Orleans, USA. ACM, January 2003.

3. N. Berregeb, A. Bouhoula, and M. Rusinowitch. SPIKE-AC: A system for proofs by induction in associative-commutative theories. In Rewriting Techniques and Applications, RTA'96, pages 428-431, 1996.

4. A. Bouhoula, E. Kounalis, and M. Rusinowitch. SPIKE: An automatic theorem prover. In Proceedings of the 1st International Conference on Logic Programming and Automated Reasoning, St. Petersburg (Russia), volume 624 of Lecture Notes in Artificial Intelligence, pages 460-462, July 1992.

5. A. Bundy, D. Basin, D. Hutter, and A. Ireland. Rippling: Meta-Level Guidance for Mathematical Reasoning. Cambridge University Press, 2005.

6. H. Comon and R. Nieuwenhuis. Induction=i-axiomatization+first-order consistency. Inf. Comput., 159(1-2):151-186, 2000.

7. E. Deplagne. Système de preuve modulo récurrence. Thèse de doctorat, Université Nancy 1, November 2002.

8. E. Deplagne and C. Kirchner. Induction as deduction modulo. Research report A04-R-468, LORIA, Nov 2004.

9. E. Deplagne, C. Kirchner, H. Kirchner, and Q.-H. Nguyen. Proof search and proof check for equational and inductive theorems. In F. Baader, editor, Proceedings of $C A D E-19$, Miami, Florida, July 2003. Springer-Verlag.

10. N. Dershowitz and D. A. Plaisted. Rewriting. In A. Robinson and A. Voronkov, editors, Handbook of Automated Reasoning, volume I, chapter 9, pages 535-610. Elsevier Science, 2001.

11. G. Dowek. La part du Calcul. Université de Paris 7, 1999. Mémoire d'habilitation.

12. G. Dowek, T. Hardin, and C. Kirchner. HOL- $\lambda \sigma$ an intentional first-order expression of higher-order logic. Mathematical Structures in Computer Science, 11(1):2145, 2001.

13. G. Dowek, T. Hardin, and C. Kirchner. Theorem proving modulo. Journal of Automated Reasoning, 31(1):33-72, Nov 2003. 
14. M. Ferreira. Termination of Term Rewriting: Well foundedness, Totality and Transformations. PhD thesis, Utrecht University, 1995.

15. H. Ganzinger and J. Stuber. Inductive theorem proving by consistency for firstorder clauses. In M. Rusinowitch and J.-L. Rémy, editors, Conditional Term Rewriting Systems, Third International Workshop, LNCS 656, pages 226-241, Pont-à-Mousson, France, July 8-10, 1992. Springer-Verlag. Published in 1993.

16. J.-Y. Girard, Y. Lafont, and P. Taylor. Proofs and Types, volume 7 of Cambridge Tracts in Theoretical Computer Science. Cambridge University Press, 1989.

17. G. Huet. Constrained Resolution: A Complete Method for Higher Order Logic. PhD thesis, Case Western Reserve University, 1972.

18. J.-M. Hullot. Canonical forms and unification. In Proceedings 5th International Conference on Automated Deduction, Les Arcs (France), pages 318-334, July 1980.

19. D. Kapur and H. Zhang. An overview of rewrite rule laboratory (RRL). J. Computer and Mathematics with Applications, 29(2):91-114, 1995.

20. C. Kirchner and H. Kirchner. Rewriting, solving, proving. A preliminary version of a book available at www.loria.fr/ ckirchne/rsp.ps.gz, 1999.

21. C. Kirchner, H. Kirchner, and M. Rusinowitch. Deduction with symbolic constraints. Revue d'Intelligence Artificielle, 4(3):9-52, 1990. Special issue on Automatic Deduction.

22. D. Musser. On proving inductive properties of abstract data types. In Proceedings, Symposium on Principles of Programming Languages, volume 7. Association for Computing Machinery, 1980.

23. F. Nahon. Preuve par induction dans le calcul des séquents modulo. $\mathrm{PhD}$ thesis, Université Henri Poincaré - Nancy I, Nancy, France, October, 262007.

24. F. Nahon, C. Kirchner, H. Kirchner, and P. Brauner. Inductive Proof Search Modulo. Annals of Mathematics and Artificial Intelligence, 55(1-2):123-154, 02 2009.

25. G. Steel. Proof by consistency - a literature survey, mar 1999.

26. B. Wack. Typage et déduction dans le calcul de réécriture. Thèse de doctorat, Université Henri Poincaré - Nancy I, October, 7- 2005. 